\title{
The occurrence of osteosarcoma after tibial fracture repair in a dog
}

\author{
Mitsuhiro Isaka*, Daiki Kokubo and Toshikazu Sakai
}

Department of Small Animal Clinical Sciences, School of Veterinary Medicine, Rakuno Gakuen University, Ebetsu, Japan

\begin{abstract}
Background: There are few detailed reports on implant-associated sarcoma in dogs; however, loose implants, metal type, and infection have not been shown as specific risk factors for this condition.

Case Description: A 14-year-old spayed female Labrador retriever was referred to our hospital with a main complaint of chronic right hind lameness after previous tibial fracture repair. On radiographs, rupture of the bone plate and screws with swelling of the surrounding soft tissue was observed, and osteosarcoma (OSA) was diagnosed after histopathological examinations. During amputation surgery, a plastic band was found associated with the implant.

Conclusion: Veterinary surgeons should be aware of implant-associated OSA and refrain from using non-medical materials in the implants. Furthermore, they should recommend the removal of orthopedic implants after fracture repair.
\end{abstract}

Keywords: Dogs, Fracture, Implant, Osteosarcoma, Repair.

\section{Introduction}

Osteosarcoma (OSA) is a highly aggressive mesenchymal tumor that comprises at least $85 \%$ of canine bone tumors (Egenvall et al., 2007) and tends to occur in larger individuals, and the most common site in dogs is in the appendicular skeleton near metaphyses (Morello et al., 2011). Several studies have suggested that OSAs result from abnormalities in osteoblast differentiation, as mesenchymal stem cells differentiate through osteoprogenitor and osteoblast phases before becoming mature osteocytes (Tang et al., 2008). Osteoblastic features are common in OSA, suggesting that early disruption of osteogenic differentiation may occur during sarcoma development (Tang et al., 2008). Furthermore, this process is sometimes initiated by the presence of orthopedic implants (Murphy et al., 1997; Rose et al., 2005). Loose implants, metal type, and infection have not been shown to be specific risk factors of implant-associated sarcoma (Sinibaldi et al., 1976; Kenecht et al., 1978; MacDonald et al., 2012). There have been a few reports on implant-associated sarcoma in dogs, albeit with minimal information (Burton et al., 2015; Arthur et al., 2016). Our case describes canine implant-associated OSA at the site of a previous fracture, repaired with bone plate and screws and an unknown plastic band.

\section{Case Details}

A 14-year-old spayed female Labrador retriever weighing $21.3 \mathrm{~kg}$ was referred to our hospital with a main complaint of chronic right-hind lameness. This patient had undergone surgery for right tibial fracture repair with implantation of bone plate and screws at a referral hospital 12 years ago. The patient showed no appetite, was not drinking, and was lethargic, with a body condition score of $1 / 5$. The patient was unable to stand up on her own, her right hind limb was mostly weak, and she was unable to move independently using her left side. The patient's state of consciousness was normal. On physical examination, her vital signs were normal (temperature: $38.1^{\circ} \mathrm{C}$, pulse 104 beats/minutes with no heart murmur, respiratory rate with normal lung sounds: 32 beats/minutes). The mucous membrane showed a pale color because of severe anemia (packed cell volume $20.4 \%$ ). In addition, the capillary refill time was delayed by $<3$ seconds, suggesting severe dehydration. The patient showed swelling and was warm to the touch from the right tibia to the femur, with a swollen right popliteal lymph node, which presented with a ruptured mass lesion approximately $7 \times 10 \mathrm{~cm}$ in size. The thoracic radiograph was normal (Fig. 1). However, the right hind limb radiograph showed an osteolytic lesion accompanying the ruptured implant and loosening of the plate and screws, with surrounding soft tissue swelling (Fig. 2). Fine needle aspiration from this site suggested OSA (data not shown). Although we could not perform angiographic computed tomography because of the owner's dissent, we performed amputation from the hip joint on the same day. Gross morphology showed severe inflammation surrounding soft tissues, the rupture of the stainless-steel bone plate, and we noted a non-medical band at the right tibia (Fig. $3)$. The patient was formally diagnosed with OSA after histopathological examinations (Fig. 4). After 24 hours hospitalization, we administered ampicillin potassium (20 mg/kg, BID) intravenously and prednisolone (0.5 $\mathrm{mg} / \mathrm{kg}$, SID) subcutaneously for postoperative care. Also, 24 hours continuous rate infusion of fentanyl

*Corresponding Author: Mitsuhiro Isaka. Department of Small Animal Clinical Sciences, School of Veterinary Medicine, Rakuno Gakuen University, Ebetsu, Japan. Email: m-isaka@rakuno.ac.jp 

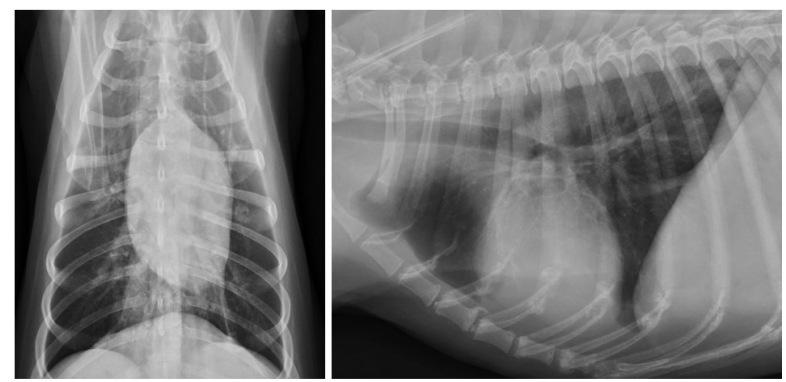

Fig. 1. Thoracic radiographs.
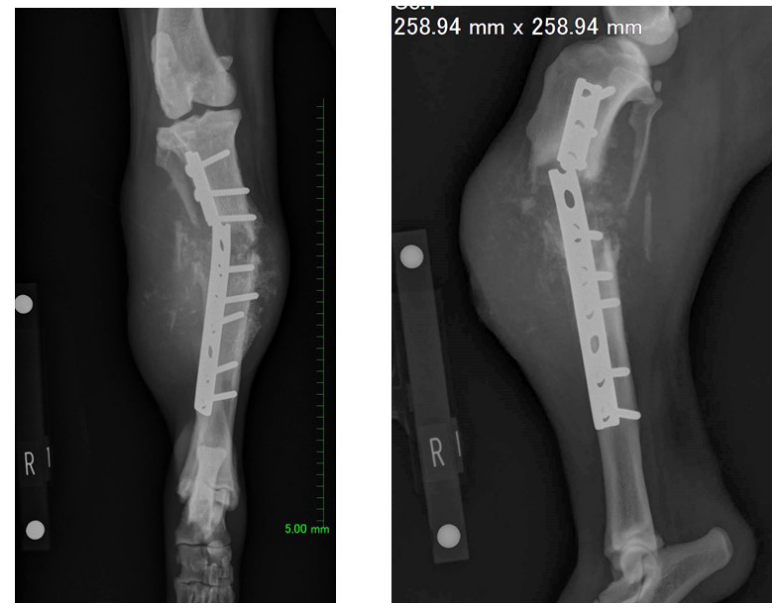

No metastasis was observed.

Fig. 2. Limb radiographs. The right tibia showed an osteolytic lesion with rupture of orthopedic plate, loosening of screws, and also soft tissue swelling.

( $5 \mu \mathrm{g} / \mathrm{kg} / \mathrm{minutes}$ ) in lactated Ringer's solution was used for analgesia. The patient was discharged to the owner the day after surgery for home care. The owner refused postoperative radiation therapy and chemotherapy. Unfortunately, this patient died 1 month after amputation.

\section{Discussion}

Orthopedic implants are commonly used in small animal patients, including dogs, and hence an awareness of potentially serious complications is important. In a study of more than 19,000 open fracture restoration cases in canines occurring between 1970 and 2000, only 15 cases of OSA had occurred at the site of fracture restoration. In other words, the incidence of OSA at a fracture site was less than $0.08 \%$ (Arthur et al., 2016). Although long-term inflammation is recognized as a contributing factor to creating an environment promoting tumorigenesis, this relationship has not been demonstrated in reports of implant-associated malignancy. While the effects of implant composition and wear have received great attention, the role of chronic inflammation in the development of sarcoma

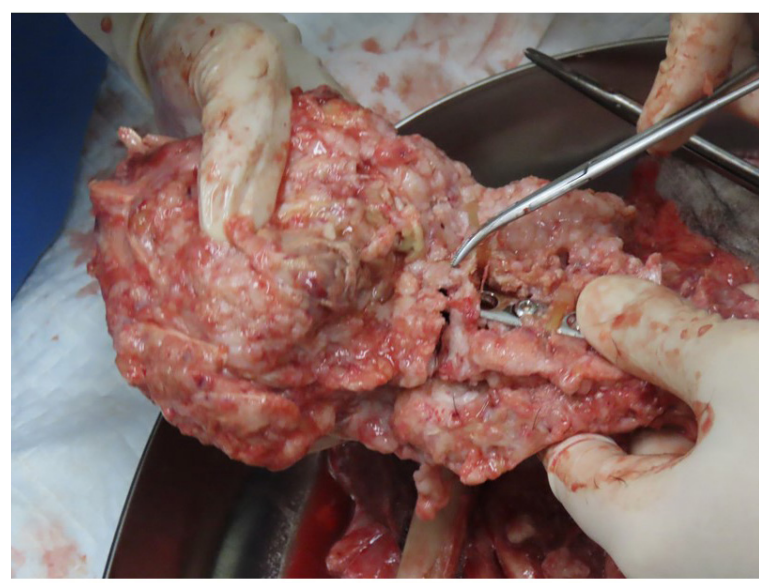

Fig. 3. An unusual plastic band was found around the orthopedic plate.

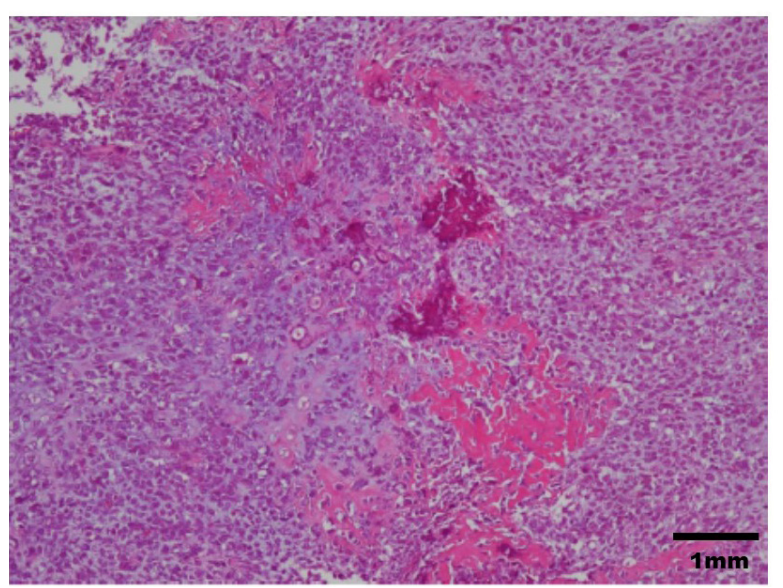

Fig. 4. Histopathological diagnosis was OSA.

remains a point of interest (Visuri et al., 2006). Many initiating factors have been hypothesized to play a role in the development of implant-associated sarcoma. Researchers have shown that many implant materials, including commonly used stainless steel and titanium, have potentially carcinogenic properties (Kirkpatrick et al., 2000). Consequently, some surgeons suggest the removal of orthopedic implants after fracture repair. In our case, non-medical bands had been used to surround the bone plate, probably leading to severe inflammation and resulting in the occurrence of OSA at the fracture site. In veterinary medicine, there are few reports about canine implant-associated neoplasia (Burton et al., 2015; Arthur et al., 2016). In a retrospective study of 16 cases of canine implant-associated tumors, it was determined that tumors developed an average of 5.5 years after implant placement. In addition, the hind limbs [tibia (8/16) and femur (5/16)] were favored; 15 of the 16 cases developed in the bone trunk and 13 were OSAs (Burton et al., 2015). Our case showed the same 
features as those documented in the previous report (Burton et al., 2015).

Although the interaction between vinyl chloride and OSA has not been reported in dogs, various chemicals (fluoride, beryllium, and vinyl chloride), exposure to radiation, and viruses have been identified as factors, inducing OSA, in humans (Operskalski et al., 1987). The patient in this case report did not have radiation therapy, no medical record of virus infection, or family history of OSA. Hence, we concluded that the vinyl chloride bands might be associated with the development of OSA.

In summary, veterinary surgeons should be able to recognize implant-associated OSA and should be discouraged from using non-medical materials in implants. Furthermore, we recommend the removal of orthopedic implants after fracture repair.

\section{Conflict of interest}

The authors declare that there is no conflict of interest. Authors' contributions

Mitsuhiro Isaka, Daiki Kokubo, and Toshikazu Sakai conceived and performed the procedure, drafted and revised the manuscript, and approved the final version.

\section{References}

Arthur, E.G., Arthur, G.L., Keeler, M.R. and Bryan, J.N. 2016. Risk of osteosarcoma in dogs after open fracture fixation. Vet. Surg. 45, 30-35.

Burton, A.G., Johnson, E.G., Vernau, W. and Murphy, B.G. 2015. Implant-associated neoplasia in dogs: 16 cases (1983-2013). J. Am. Vet. Med. Assoc. 247, 778-785.

Egenvall, A., Nodtvedt, A. and von Euler, H. 2007. Bone tumors in a population of 400000 insured Swedish dogs up to $10 \mathrm{y}$ of age: incidence and survival. Can. J. Vet. Res. 71, 292-299.
Kenecht, C.D. and Priester, W.A. 1978. Osteosarcoma in dogs: a study of previous trauma, fracture and fracture fixations. J. Am. Anim. Hosp. Assoc. 14, 82-84.

Kirkpatrick, C.J., Alves, A. and Köhler, H., Kriegsmann, J., Bittinger, F., Otto, M., Williams, D.F. and Eloy. 2000. Biomaterial-induced sarcoma: a novel model to study preneoplastic change. Am. J. Pathol. 156, 1455-1467.

MacDonald, D.J., Enneking, W.F. and Sundaram, M. 2002. Metal-associated angiosarcoma of bone: report of two cases and review of the literature. Clin. Orthop. Relat. Res. 396, 206-214.

Morello, E., Martano, M. and Buracco, P. 2011. Biology, diagnosis and treatment of canine appendicular osteosarcoma: similarities and differences with human osteosarcoma. Vet. J. 89, 268-277.

Murphy, S.T., Parker, R.B. and Woodard, J.C. 1997. Osteosarcoma following total hip arthroplasty in a dog. J. Small. Anim. Pract. 38, 263-267.

Operskalski, E.A., Martin, S.P., Henderson, B. and Vicsher, B, R. 1987. A case control study of osteosarcoma. Am. J. Epidemiol. 126, 118-119.

Rose, B.W., Novo, R.E. and Olson, E.J. 2005. Osteosarcoma at the site of a triple pelvic osteotomy in a dog. J. Am. Anim. Hosp. Assoc. 41, 327-331.

Sinibaldi, K., Rosen, H. and Liu, S.K. and DeAngelis, M. 1976. Tumor associated with metallic implants in animals. Clin. Orthop. 118, 257-266.

Tang, N., Song, W.X. and Luo, J., Haydon, R.C. and He, TC. 2008. Osteosarcoma development and stem cell differentiation. Clin. Orthop. Relap. Res. 466, 2114-2130.

Visuri, T., Pulkkinen, P. and Paavolainen P. 2006. Malignant tumors at the site of total hip prosthesis. Analytic review of 46 cases. J. Arthroplasty. 21, 311-323. 